\title{
Outcomes of in-hospital treatment of cardiac patients who survived cardiac arrest and experienced coronary angiography
}

\author{
Mindaugas Barauskas, Ramūnas Unikas, Greta Žiubrytė
}

Department of Cardiology, Lithuanian University of Health Sciences, Kaunas, Lithuania

Submitted: 29 November 2017

Accepted: 15 January 2018

Arch Med Sci Atheroscler Dis 2018; 3: e1-e7

DOI: https://doi.org/10.5114/amsad.2018.73212

Copyright @ 2018 Termedia \& Banach

\section{Abstract}

Introduction: As coronary artery disease is the most frequent cause of cardiac arrest, early invasive strategies may be beneficial for such patients. This study analyses the impact of in-hospital treatment on short-term outcomes of out-of-hospital cardiac arrest (OHCA) survivors.

Material and methods: Patients admitted to the Cardiac Intensive Care Unit of our hospital within 2-year period were prospectively included in the study. Results: One hundred thirty-one patients were included in the study, which showed that in-hospital mortality increases uniformly with the severity of the coronary artery lesion ( $p=0.044)$, but an effect of revascularization on number of deaths was not observed $(p=0.64)$. The presence of coma $(p=0.005)$ and the combination of male sex and age above 60-year as 2.2-fold $(p=0.048)$ increasing in-hospital mortality were found. The highest mortality rate occurred during the first 3 days and the death rate of the patients who survived this period is low. We found reduced left ventricular ejection fraction $(O R=$ 6.54; $95 \% \mathrm{Cl}: 1.98-21.63 ; p=0.002)$, non-ventricular fibrillation initial rhythm $(\mathrm{OR}=2.94 ; 95 \% \mathrm{Cl}: 1.25-6.90 ; p=0.014)$, unconscious at admission (OR $=6.46 ; 95 \% \mathrm{Cl}: 1.96-21.24 ; p=0.002)$ and post-resuscitation coma $(\mathrm{OR}=$ $6.00 ; 95 \% \mathrm{Cl}: 2.63-13.66 ; p<0.001)$ or encephalopathy $(\mathrm{OR}=2.71 ; 95 \% \mathrm{Cl}$ : $1.9-6.72 ; p=0.031$ ) to be significant prognostic factors for higher in-hospital mortality rate.

Conclusions: We recommend immediate coronary interventions for all survivors of OHCA regardless of their state of consciousness and absence of ischaemic changes on ECG. Early intensive treatment for OHCA patients is indispensable, as the highest mortality rate is within the first 3 days after an event.

Key words: acute cardiac care, coronary interventions, invasive treatment, myocardial infarction.

\section{Introduction}

Cardiac arrest is a common cause of death with particularly high rates of fatality and disability. More than three quarters of cardiac arrests occur out of hospital $[1,2]$. As ischaemic heart disease is the most common cause of out-of-hospital cardiac arrest (OHCA) [1-3] immediate coronary artery angiography (CAG) is recommended as the first line diagnostic method for such patients despite absence of ischaemic changes on electrocardiography (ECG) [1, 3]. Patients with significant coronary artery lesions who experienced successful percutaneous coronary intervention $(\mathrm{PCl})$ have better outcomes compared to those who did not undergo $\mathrm{PCl}$

\author{
Corresponding author: \\ Mindaugas Barauskas \\ Department of Cardiology \\ Lithuanian University \\ of Health Sciences \\ 2 Eiveniu St \\ 50009 Kaunas, Lithuania \\ Phone: +37 068625822 \\ E-mail: mbarauskas101@ \\ hotmail.com
}


or in whom the procedure was not successful [2]. Although there is a class I level $B$ recommendation for management of OHCA survivors with ST elevation on ECG in guidelines published in 2017 by the European Society of Cardiology, management of non-ST-segment myocardial infarction (NSTEMI) OHCA survivors remains a complex issue that has been poorly studied worldwide [4].

Some studies define therapeutic hypothermia $(\mathrm{TH})$ to be alternative treatment for OHCA patients [5]. There is some evidence that $\mathrm{TH}$ as complementary management of OHCA surviving patients who presented unconscious has a superior effect on survival and neurological status [2, $4,6,7]$. North American researchers have suggested that early CAG and TH provide better survival and overall functional status for OHCA patients [6]. The American Academy of Neurology strongly supports $\mathrm{TH}$ for superior neurological recovery in OHCA patients [7]. Despite that, data regarding the impact of $\mathrm{TH}$ on improved in-hospital survival in OHCA victims are scarce.

The aim of this paper is to discuss our experience in the context of observational studies, meta-analyses and guidelines recommendations on in-hospital management and short-term outcomes for survivors of OHCA.

\section{Material and methods}

\section{Study population}

Patients admitted to the Cardiac Intensive Care Unit (CICU) in the Hospital of Lithuanian University of Health Sciences Kaunas Clinics, Kaunas, Lithuania between March 2012 and January 2014 were prospectively included in the study. All included patients had survived cardiac arrest, been resuscitated and presented to our Department of Cardiology whether conscious or unconscious. There were no exclusion criteria for patients who survived OHCA with suspected cardiac origin cardiac arrest. All patients were included in the study after their or their relatives' written consent was given. Local Ethics Committee approval was obtained for the study.

\section{Study protocol}

The patients' baseline demographic (age, sex,) and functional status (Glasgow Coma Scale (GCS) score, heart rate (HR), blood pressure (BP), ECG at admission, potassium level in plasma, comorbidities, findings of CAG and echocardiography examinations and short-term outcomes were observed during the in-hospital period.

\section{Coronary artery angiography}

Coronary artery angiography was performed within $6 \mathrm{~h}$ of admission for all patients, while it was performed immediately (within $60 \mathrm{~min}$ ) for patients with evident ST segment elevation on ECG. Percutaneous coronary intervention was attempted immediately and dual antiplatelet therapy started if the culprit lesion could be identified.

\section{Echocardiography}

Echocardiographic analysis of heart dimensions, left ventricular dysfunction and valvular abnormalities was performed during the first $24 \mathrm{~h}$ of admission.

Wall motion index (WMI) was acquired in the apical two-chamber view by use of a 16-segment model, where 1 means normal myocardial contraction and the higher the number the poorer contraction of the segment.

Left ventricular ejection fraction (LVEF) was measured in the apical two- and four-chamber views by tracking the endocardium at the end of both systole and diastole in both views, calculated by Simpson's method following the computed formula.

The presence of cardiomyopathy and mild or severe regurgitation or stenosis of the heart valves were taken into consideration by overall cardiac function evaluation.

\section{Therapeutic hypothermia}

Therapeutic hypothermia was applied at the time of admission. The target temperature between 32 and $36^{\circ} \mathrm{C}$ was maintained for $24 \mathrm{~h}$ by cold intravenous fluids.

\section{Statistical analysis}

The statistical analysis was performed using the software package SPSS 20.0 (IBM Corp., Armonk, NY, USA). All quantitative data were expressed as mean \pm standard deviation ( \pm SD). Sample values were compared using Student's paired t-test. Two dependent variables were assessed by Pearson's correlation and test. Correlation of nominal data was evaluated by the $\chi^{2}$ test. The Cox proportional-hazards regression model was chosen for survival analysis. The chosen significance level was $p<0.05$.

\section{Results}

\section{Patients' baseline demographic and clinical characteristics}

The age of the study population varies from 29 to 91 years with mean age of $62 \pm 11$.7. Ninety-six of 131 post-cardiac arrest patients included in the analysis were males with mean age of $61 \pm 11.4$. Mean age of 35 female patients was $64.9 \pm 12.2$.

Patients' clinical characteristics at admission are shown in Table I. $28.2 \%(36 / 131)$ of the study patients had the hypokalaemia (a lower than normal plasma potassium level $(<3.5 \mathrm{mmol} / \mathrm{l}))$ and 
Table I. Clinical characteristics and medical history of the study population

\begin{tabular}{|c|c|c|}
\hline Clinical characteristic & \multicolumn{2}{|c|}{ Percent (number of cases) of study population } \\
\hline Unconscious (Glasgow Coma Scale score 3-9 points) & \multicolumn{2}{|c|}{$66.4 \%(87 / 131)$} \\
\hline Conscious & \multicolumn{2}{|c|}{$33.6 \%(44 / 131)$} \\
\hline Clinical characteristic & $\begin{array}{c}\text { Mean value } \\
\text { (standard deviation) }\end{array}$ & $\begin{array}{c}\text { Value range } \\
\text { (min.-max. value) }\end{array}$ \\
\hline Heart rate [bpm] & $89.4 \pm 22.1$ & $46-190$ \\
\hline Systolic blood pressure [mm Hg] & $126.1 \pm 31.3$ & $50-215$ \\
\hline Diastolic blood pressure [mm Hg] & $77.5 \pm 17.5$ & $30-117$ \\
\hline Plasma potassium level [mmol/l] & $3.87 \pm 0.7$ & $2.4-6.1$ \\
\hline STEMI on ECG & \multicolumn{2}{|l|}{$55 \%(72 / 131)$} \\
\hline Medical condition & \multicolumn{2}{|c|}{ Percent (number of cases) of study population } \\
\hline $\mathrm{AH}$ & \multicolumn{2}{|c|}{$73.3 \%(96 / 131)$} \\
\hline Dyslipidaemia & \multicolumn{2}{|c|}{$53.4 \%(70 / 131)$} \\
\hline MI & \multicolumn{2}{|c|}{$19.1 \%(25 / 131)$} \\
\hline Metabolic syndrome & \multicolumn{2}{|c|}{$13 \%(17 / 131)$} \\
\hline Stroke and transient ischaemic attack & \multicolumn{2}{|c|}{$9.2 \%(12 / 131)$} \\
\hline DM & \multicolumn{2}{|c|}{$9.2 \%(12 / 131)$} \\
\hline $\mathrm{PCl}$ & \multicolumn{2}{|c|}{$10.7 \%(14 / 131)$} \\
\hline Coronary artery bypass graft surgery & \multicolumn{2}{|c|}{$6.1 \%(8 / 131)$} \\
\hline Cardiac arrhythmias & \multicolumn{2}{|c|}{$7.6 \%(10 / 131)$} \\
\hline Oncology & \multicolumn{2}{|c|}{$6.1 \%(8 / 131)$} \\
\hline Peripheral artery disease & \multicolumn{2}{|c|}{$3.1 \%(4 / 131)$} \\
\hline
\end{tabular}

STEMI - ST segment elevation myocardial infarction, ECG - electrocardiogram, AH - arterial hypertension, MI - myocardial infarction, $D M$ - diabetes mellitus, $P C I$ - percutaneous coronary intervention.

$3.8 \%(5 / 131)$ of all patients had the hyperkalaemia (a higher than normal plasma potassium level $(>5.1 \mathrm{mmol} / \mathrm{l}))$. The remaining $67.9 \%(89 / 131)$ of patients had a normal plasma potassium level $(3.5-5.1 \mathrm{mmol} / \mathrm{l})$.

\section{Patients' medical history and causes of cardiac arrest}

In patients' medical history, arterial hypertension $(\mathrm{AH})$, dyslipidaemia, myocardial infarction (MI), metabolic syndrome (MS) and diabetes mellitus (DM) were the most frequent. The prevalence of all comorbidities is shown in Table I. A history of smoking was noted in $32.8 \%$ (43/131) of cases.

Predominant causes of cardiac arrest were ventricular fibrillation (VF), which occurred in $92.4 \%$ $(122 / 131)$ of patients. Asystole and electromechanical dissociation (EMD) each occurred in 3.8\% (5/131) of patients.

Post-resuscitation neurological impairment presented in $43.5 \%(57 / 131)$ of cases, $18.3 \%$ $(24 / 131)$ and $25.2 \%(33 / 131)$ of which were post-anoxic coma and post-anoxic encephalopathy, respectively. The neurological status had a great impact on short-term outcomes. Patients who experienced post-anoxic coma or post-anoxic encephalopathy had $25.8(p<0.001)$ or 5.7 ( $p=$ 0.002 ) times higher risk for death, respectively.

\section{Coronary artery angiography and revascularization strategies}

Post-cardiac arrest CAG was performed for all 131 patients within $6 \mathrm{~h}$ of admission. The revascularization strategy was chosen in accordance with the findings of CAG (Table II). 24.4\% (32/131) of patients did not undergo any revascularization, 63.4\% (83/131) had CAG followed by ad hoc PCI of one coronary artery, and $\mathrm{PCl}$ of two or three arteries was performed in 9.2\% (12/131) and 1.5\% (2/131) cases, respectively. Percutaneous coronary interventions of the left main coronary artery (LMCA) was performed in $1.5 \%(2 / 131)$ of cases and $3.1 \%(4 / 131)$ of patients were sent for coronary artery bypass graft surgery (CABG). 
Table II. Coronary artery angiography findings in study population

\begin{tabular}{|lc|}
\hline $\begin{array}{l}\text { Coronary artery } \\
\text { angiography findings }\end{array}$ & $\begin{array}{r}\text { Percent (number of cases) } \\
\text { of study population }\end{array}$ \\
\hline Normal coronary arteries & $12.2 \%(16 / 131)$ \\
\hline One-vessel disease & $25.9 \%(34 / 131)$ \\
\hline Two-vessel disease & $21.4 \%(28 / 131)$ \\
\hline Three-vessel disease & $41.2 \%(54 / 131)$ \\
\hline $\begin{array}{l}\text { Left main coronary artery } \\
\text { disease }\end{array}$ & $6.9 \%(9 / 131)$ \\
\hline
\end{tabular}

\section{Echocardiographic test results}

Hypertrophic cardiomyopathy and heart valve dysfunction were diagnosed in $35.1 \%(46 / 131)$ and $6.9 \%(9 / 131)$ of cases within $24 \mathrm{~h}$ after admission, respectively. Left ventricular ejection fraction varies from 5 to $55 \%$ with a mean value of $34.6 \pm 11.9$ and $W M I$ varies from 1.06 to 2.53 with a mean value of $1.82 \pm 0.37$.

\section{In-patient management and final diagnosis}

Time from event to admission varies from 8 to 2880 min with a mean value of $138.2 \pm 299.4$. In-patient treatment duration varies from 1 to 190 days with a mean of $13.3 \pm 18.6$ days.

Final diagnosis of the patients' is shown in Table III.

\section{Short-term outcomes}

$66.4 \%(87 / 131)$ of all patients have recovered, $3.8 \%(5 / 131)$ were referred to another hospital and 29.8\% (39/131) died. Of those 39 patients who died $22.1 \%$ (29/131) were male and $7.6 \%(10 / 131)$ were female. There were no significant mortality differences between the groups $(p>0.05)$.

The hyperkalaemia increased the risk of death for survivors of OHCA 8.23 times ( $p=0.04)$.

Patients with significant LMCA disease (> 50\% obstruction) had 3.01 times higher risk of dying within the in-hospital period $(p=0.26)$. None of such patients have survived in our study.

LVEF has a significant impact on in-hospital survival $(p=0.001)$. Patients with LVEF less than $40 \%$ had higher risk for in-hospital mortality. WMI higher than 1.72 results in 5.6 times greater in-hospital mortality risk $(p=0.025)$.

$\mathrm{TH}$ as an additional treatment method was applied to $16.8 \%$ (22/131) of patients, 7 of whom were comatose at admission. $10.7 \%$ (14/131) of the whole study population died before hospital discharge, representing $63.6 \%(14 / 22)$ of patients for whom TH was applied. The mortality rate for non-TH patients was $18.4 \%$ (20/109) in our study.
Table III. Diagnosis at discharge of study population

\begin{tabular}{|lc|}
\hline Final diagnosis & $\begin{array}{c}\text { Percent (number of cases) } \\
\text { of study population }\end{array}$ \\
\hline STEMI & $55 \%(72 / 131)$ \\
\hline NSTEMI & $30.5 \%(40 / 131)$ \\
\hline Unstable angina & $0.8 \%(1 / 131)$ \\
\hline $\begin{array}{l}\text { Chronic coronary artery } \\
\text { disease }\end{array}$ & $0.8 \%(1 / 131)$ \\
\hline Other causes & $13.7 \%(18 / 131)$ \\
\hline $\begin{array}{l}\text { STEMI - ST segment elevation myocardial infarction, NSTEMI - } \\
\text { non-ST segment elevation myocardial infarction. }\end{array}$
\end{tabular}

We did not define $\mathrm{TH}$ to reduce the number of deaths before hospital discharge. Furthermore, in our study TH seems to cause more adverse effects such as pneumonia.

Our study has shown that in-hospital mortality increases uniformly with the severity of the CA lesion ( $p=0.044$ ), but an effect of revascularization on number of deaths was not revealed $(p=0.64)$. This study has found that presence of coma $(p=0.005)$ and age over 60 years $(p=0.07)$ significantly increase short-term mortality. The combination of male sex and age above 60 years was found to be a factor increasing the in-hospital mortality 2.2 times $(p=0.048)$ in the present study. In addition, we concluded that the highest mortality is during the first 3 days and the death rate of the patients who survived this period is low $(p<0.001)$. 30-day in-hospital mortality data are shown in Figure 1. On unmentioned days, no patient died. We observed the highest rate of mortality during the first 3 days, when 22 of 131 (16.8\%) patients died; of these the most critical is the first day $(11 / 131$ (8.4\%) patients died on the first day). 7-day and 14-day mortality are additionally 7/131 (plus 5.34\%) and $3 / 131$ (plus $2.3 \%$ ) death cases, respectively. 30-day mortality adds 6/131 (plus 4.6\%) death cases.

\section{Survival analysis}

In a univariate analysis hypokalaemia, LVEF lower than $40 \%$, initial rhythm defined as nonVF (this group consisted of asystole and EMD) and neurological status at admission and after resuscitation (post-anoxic coma and post-anoxic encephalopathy) were found to be significant prognostic factors for lower 30-day survival rate compared to opposite groups (Table IV). Furthermore, the combination of LVEF lower than $40 \%$, non-VF initial rhythm, unconscious at admission and post-anoxic coma after resuscitation were found to be significant prognostic factors of poor survival to hospital discharge in a multivariate analysis (Table IV). 


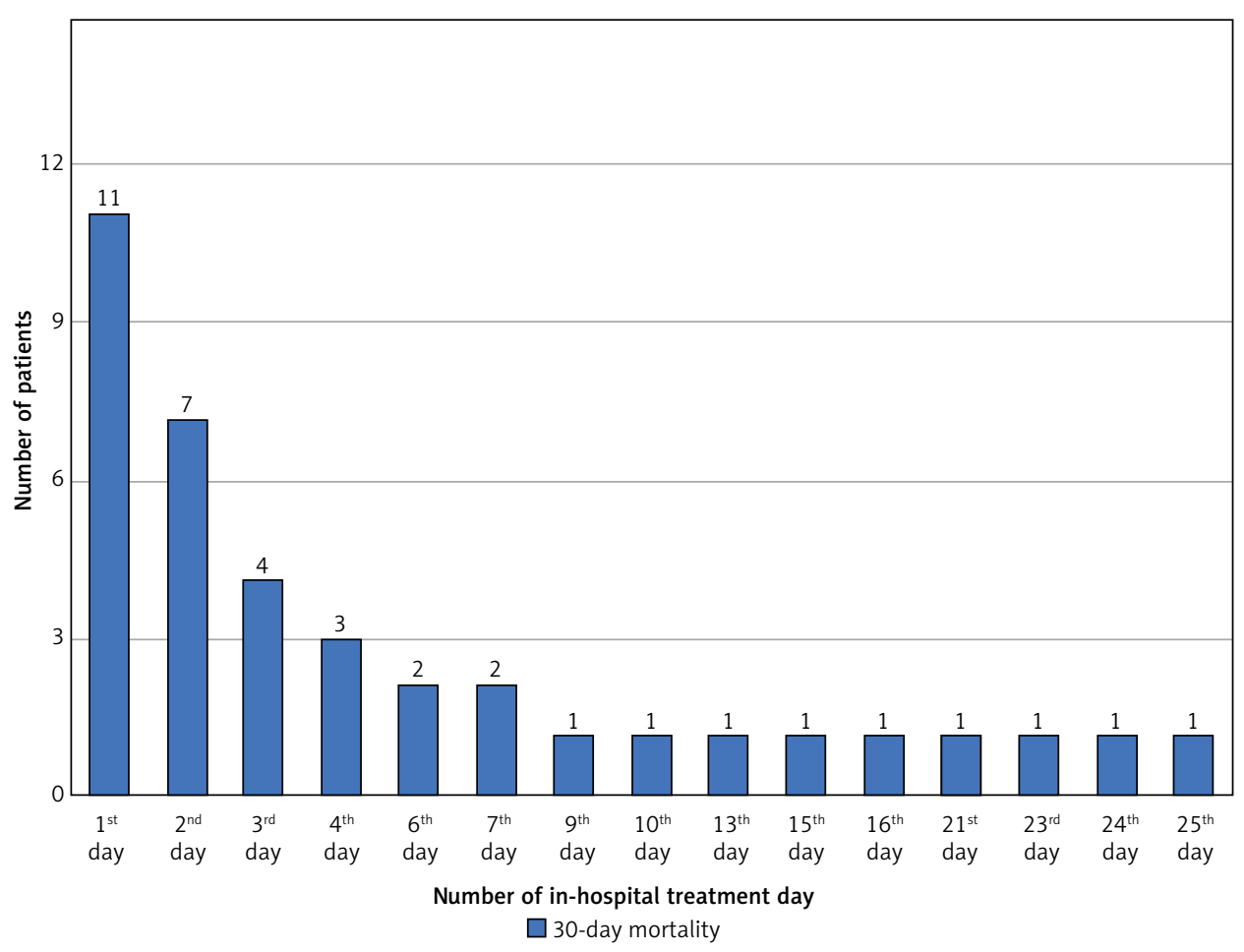

Figure 1. 30-day in-hospital mortality

Table IV. Univariate and multivariate prognostic factors of 30-day survival after OHCA

\begin{tabular}{|c|c|c|c|c|c|c|}
\hline \multirow[t]{2}{*}{ Variable } & \multicolumn{3}{|c|}{ Univariate analysis } & \multicolumn{3}{|c|}{ Multivariate analysis } \\
\hline & OR & $95 \% \mathrm{Cl}$ & $P$-value & OR & $95 \% \mathrm{Cl}$ & $P$-value \\
\hline $\begin{array}{l}\text { Plasma potassium level: } \\
3.5-5.1 \mathrm{mmol} / \mathrm{l} \text { vs. }<3.5 \mathrm{mmol} / \mathrm{l}^{a}\end{array}$ & 0.44 & $0.19-1.03$ & 0.050 & 0.26 & $0.39-7.14$ & 0.490 \\
\hline LVEF: $>40 \%$ vs. $<40 \%^{a}$ & 6.54 & $1.98-21.63$ & 0.002 & 13.56 & $1.41-130.72$ & 0.024 \\
\hline Initial rhythm: VF vs. non-VFa & 2.94 & $1.25-6.90$ & 0.014 & 0.36 & $0.05-2.66$ & 0.316 \\
\hline $\begin{array}{l}\text { Post-resuscitation neurological } \\
\text { status: normal vs. postanoxic } \\
\text { coma }\end{array}$ & 6.00 & $2.63-13.66$ & $<0.001$ & 40.18 & $3.94-409.73$ & 0.002 \\
\hline $\begin{array}{l}\text { Post-resuscitation neurological } \\
\text { status: normal vs. postanoxic } \\
\text { encephalopathy }\end{array}$ & 2.71 & $1.09-6.72$ & 0.031 & 1.68 & $0.39-7.14$ & 0.490 \\
\hline $\begin{array}{l}\text { Neurological status at admission: } \\
\text { conscious vs. unconscious }{ }^{a}\end{array}$ & 6.46 & $1.96-21.24$ & 0.002 & 11.06 & $1.13-108.74$ & 0.039 \\
\hline
\end{tabular}

aReference. LVEF - left ventricular ejection fraction, VF - ventricular fibrillation, non-VF - non-ventricular fibrillation, OR - odds ratio, $\mathrm{Cl}$ - confidence interval.

\section{Discussion}

Until recently, despite the many studies conducted on STEMI following OHCA management, there was no unified agreement on NSTEMI OHCA survivors' care protocol in Europe. As approximately $40 \%$ of OHCA patients have no ST-segment elevation on ECG [8] management of NSTEMI OHCA remains an issue and a strict protocol may benefit overall survival in such cases.

As Camuglia et al. [2] in contrast to Callaway et al. [6] suggest, all patients in our study underwent immediate $\mathrm{PCl}$ regardless of the state of their consciousness and changes on ECG. The PCI rate (100\% - all 131 patients underwent CAG within $6 \mathrm{~h})$ in our study is higher than in most recent studies (11\% [8] - 73\% [9]). CAD in post-cardiac arrest NSTEMI patients was found more frequently in the current study (88.2\%) compared to most of the published data $(21-58 \%)[6,10,11]$. As many data $[2,4,6,8,11-13]$ support invasive strategies for all, even NSTEMI OHCA survivors, we may presume that urgent CAG and $\mathrm{PCl}$, when indicated, has a significant impact on superior survival to 
hospital discharge. Geri et al. proved that early CAG even not followed by PCI has no harmful effect on outcomes for patients with no indications for $\mathrm{PCl}$. Moreover, the same study declared early PCI to be associated with superior long-term survival in OHCA victims [13]. Due to evidence reported in discussed studies and our experience, we strongly support early invasive strategies for all OHCA patients with suspected cardiac origin.

As OHCA requires various treatment, some studies support combination of $\mathrm{TH}$ and $\mathrm{PCl}[4,6$, 7]. Furthermore, Kleissner et al. prefer $\mathrm{PCl}$ after recovery post $\mathrm{TH}$ [5]. Nevertheless, according to the "2017 ESC Guidelines for the management of acute myocardial infarction in patients presenting with ST-segment elevation" [14], TH should not delay CAG and $\mathrm{PCl}$ in OHCA survivors [14]. Due to scarce application of $\mathrm{TH}$ in our practice we are not able to make a recommendation for $\mathrm{TH}$ acceptance in everyday practice.

While many studies define $\mathrm{TH}$ to have a significant impact on short-term survival $[12,15]$, in consistence with Bergman et al. [11] our study did not prove induced $\mathrm{TH}$ to be associated with better outcomes after OHCA. The survival to hospital discharge rate in $\mathrm{TH}$ and non- $\mathrm{TH}$ groups was $47 \%$ vs. $56 \%$ as reported by Bergman et al. [11] compared to $8 \%$ and $92 \%$ in our study. Moreover, $\mathrm{TH}$ was associated with cold-caused complications in the current analysis. According to researchers, $\mathrm{TH}$ is prone to increase the favourable neurological recovery rate rather than overall outcome or survival to hospital discharge [15]. We did not demonstrate a survival to hospital discharge benefit of $\mathrm{TH}$, most likely due to the low number of patients in the $\mathrm{TH}$ group. For a more significant conclusion further studies with a higher number of patients are required.

Superior outcomes after OHCA in relation to the initial rhythm of ventricular fibrillation, which was dominant in our study $(92.4 \%)$, have been observed in resent studies [11, 15]. Ventricular fibrillation as an initial rhythm may add benefits to the better than average rate of survival to hospital discharge (70.2\%) in our study population, compared to other published data, where it varies between 31.8 and $37 \%[12,15]$.

Data on echocardiographic measurements in relation to outcomes after OHCA are sparse. Bergman et al. reported higher LVEF to have a significant impact on better outcomes after OHCA [11]. In addition to reduced LVEF, we may add wall motion abnormalities (WMI > 1.72) to be an important predictor factor for poor short-term survival. Those factors' impact on long-term survival requires further studies.

As neurological deficiency is reported to be the most frequent adverse outcome after resuscitation following OHCA, most authors for neurolog- ical recovery recommend combination of $\mathrm{TH}$ and early $\mathrm{PCl}$ [11]. Grasner et al. specified $\mathrm{PCl}$ to be an independent factor for a favourable neurological outcome [16]. Weiser et al. alike support an early coronary intervention strategy and prove it to be a significant index for favourable neurological recovery [13]. Our study does not evaluate neurological status at discharge, but we proved neurological deficiency to be a frequent cause of disability for post-resuscitation patients; it represented $43.5 \%$ of cases in our study. Further studies are required to prevent biases for favourable neurological recovery stratification.

The first 3 days were defined as the most critical period for survival to hospital discharge. This is consistent with the publication by Callway et al. where the first in-hospital treatment days proved to be the most critical for in-hospital deaths [6]. The reason for early mortality of OHCA survivors remains an issue, as the main Callway et al. hypothesis of death caused by STEMI was refuted by our study [6].

The main limitations of our study were the low number of patients in the TH group and the short follow-up period. Further studies with bigger samples of patients for whom TH was applied are necessary to prove or disprove benefits of $\mathrm{TH}$ application for short-term survival, and a longer follow-up period is necessary for significant stratification of the most important prognostic factors for survival after hospital discharge. Furthermore, to evaluate an early $\mathrm{PCl}$ benefit to favourable neurological status at hospital discharge, neurological status evaluation at hospital discharge is necessary.

In conclusion, we strongly recommend immediate coronary interventions for all survivors of OHCA regardless of their state of consciousness and absence of ischaemic changes on ECG. Ventricular fibrillation as an initial rhythm is a significant predictive factor for a better survival rate, while lower than $40 \%$ LVEF and wall motion abnormalities are indexes of poor outcomes in such patients. Early intensive treatment for OHCA patients is indispensable, as the highest mortality rate is within the first 3 days after an event. We conclude that LVEF lower than $40 \%$, non-ventricular fibrillation as an initial rhythm, unconscious at admission and post-anoxic coma are the most significant prognostic factors of survival after out-of-hospital cardiac arrest.

\section{Conflict of interest}

The authors declare no conflict of interest.

\section{References}

1. Jollis GJ, Granger CB. Improving care of out-of-hospital cardiac arrest. Circ J 2016; 134: 2040-2. 
2. Camuglia AC, Radhawa VK, Lavi S, Walter DL. Cardiac catheterization is associated with superior outcomes for survivors of out of hospital cardiac arrest: review and meta-analysis. Resuscitation 2014; 85: 1533-40.

3. Han SK, Park SC, Lee SH, Yeom SR, Park SW. Emergency coronary angiography in out-of-hospital cardiac arrest patients without STEMI. Am J Emerg Med 2016; 34: 118. e1-3.

4. Tanveer R, Kern KB, Tamis-Holland ET, et al. Cardiac arrest. A treatment algorithm for emergent invasive cardiac procedures in the resuscitated comatose patient. J Am Coll Cardiol 2015; 66: 62-73.

5. Kleissner M, Sramko M, Kohoutek J, Kautzner J, Kettner J. Impact of urgent coronary angiography on mid-term clinical outcome of comatose out-of-hospital cardiac arrest survivors presenting without ST-segment elevation. Resuscitation 2015; 94: 61-6.

6. Callway CW, Schimicker RH, Brown SP, et al. Early coronary angiography and induced hypothermia are associated with survival and functional recovery after out-of-hospital cardiac arrest. Resuscitation 2014; 85: 657-63.

7. Geocadin GG, Wijdicks E, Aemstrong MJ, et al. Practice guideline summary: reducing brain injury following cardiopulmonary resuscitation. Neurology 2017; 88: 2141-9.

8. Stub D, Hengel C, Chan W, et al. Usefulness of cooling and coronary catheterization to improve survival in out-of-hospital cardiac arrest. Am J Cardiol 2011; 107: 522-7.

9. Grasner JT, Meubohm P, Caliebe A, et al. Postresuscitation care with mild therapeutic hypothermia and coronary intervention after out-of-hospital cardiopulmonary resuscitation: a prospective registry analysis. Crit Care 2011; 15: R61.

10. Cronier P, Vignon P, Bouferrache K, et al. Impact of routine percutaneous coronary intervention after out-of-hospital cardia arrest due to ventricular fibrillation. Crit Care 2011; 15: R122.

11. Bergman R, Hiemstra B, Nieuwland W, et al. Long-term outcome of patients after out-of-hosptal cardiac arrest in relation to treatment: a single-centre study. Eur Heart J Acute Cardiovasc Care 2016; 5: 328-38.

12. Geri G, Dumas F, Bougouin W, et al. Immediate percutaneous coronary intervention is associated with improved short- and long-term survival after out-of-hospital cardiac arrest. Circ Cardiovasc Interv 2015; 8: e002303.

13. Weiser C, Testori C, Sterz F, et al. The effect of percutaneous coronary intervention in patients suffering from ST-segment elevation myocardial infarction complicated by out-of-hospital cardiac arrest on 30 days survival. Resuscitation 2013; 84: 602-8.

14. Ibanez B, James S, Agewall S, et al. 2017 ESC Guidelines for the management of acute myocardial infarction in patients presenting with ST-segment elevation: The Task Force for the management of acute myocardial infarction in patients presenting with ST-segment elevation of the European Society of Cardiology (ESC). Eur Heart J 2018; 39: 119-77.

15. Bosson N, Kaji AH, Niemann JT, et al. Survival and neurological outcome after out-of-hospital cardiac arrest results one year after regionalization of post-cardiac arrest care in a large metropolitan area. Prehosp Emerg Care 2014; 18: 217-23.

16. Grasner JT, Meybohm P, Caliebe A, et al. Postresuscitation care with mild therapeutic hypothermia and coronary intervention after out-of-hospital cardiopulmonary resuscitation: a prospective registry analysis. Crit Care 2011; 15R: 61. 